\title{
LAS CIUDADES PRODUCTIVAS DEL PERÚ
}

\author{
THE PRODUCTIVE CITIES OF PERU
}

Carlos Cavani Grau**
ccavanigrau@yahoo.es

[RECEPCIÓN: JULIO DEL 2014/CONFORMIDAD: SETIEMBRE DEL 2014]

De igual manera que los "burgos" en la Edad Media eran centros de actividad comercial y artesanal, las ciudades productivas y clústers representan una alternativa para generar vetas ricas en actividades económicas remuneradas generadoras de innovación, competitividad y empleo.

\section{RESUMEN}

Una "ciudad productiva" es un modelo de desarrollo socioeconómico basado en conglomerados consorcio-productivos, constituidos por cadenas de eslabonamiento productivo hacia adelante y hacia atrás o clúster, con tamaño crítico, dispuestas y organizadas cabalmente hacia las ventas y la exportación (Cavani Grau, 2009). Lo expuesto es producto de una investigación que comprueba que esto constituye una realidad palpable en el Perú, sustentada en ejemplos múltiples, donde la organización de ciudades productivas es posible a mediano y largo plazo.

La economía local de un distrito demanda múltiples servicios, oficios y negocios comerciales que hoy casi no existen. Hay, pues, una enorme necesidad de aumentar la densidad de empresas en los nuevos barrios de Lima. Y los negocios barriales generan empleo en los barrios (Infante, 2012). Las ciudades productivas están integradas por micro, pequeñas y medianas empresas. Sin embargo, sostenemos que Gamarra es un clúster incipiente como producto de la fractura estructural entre pequeñas y grandes empresas y de las debilidades estructurales que las empresas tienen en nuestro país, como también afirma Gladys Triveño.

\begin{abstract}
A productive city is a model of socio-economic development based on consortium-productive or clusters composed of chains of linkages productive front and back, with size critical, arranged and organized fully to the sales and export. Exposed - this is the product of a research - based with an extensive bibliography, as well as multiple examples that allow us to assert that the Organization of productive cities is possible in the medium and long term.
\end{abstract}

\footnotetext{
* Doctor en Administración de Negocios Globales por la Universidad Ricardo Palma y Magíster en Administración por la Universidad Nacional Mayor de San Marcos. Posee una amplia experiencia gerencial en empresas nacionales y extranjeras. Es Conferencista Internacional, Asesor de empresas y autor de varios textos en la especialidad. Actualmente es Presidente del Premio Empresa Peruana del Año, así como Presidente del Consejo Iberopanamericano de Administración.
} 
The local economy of a district demand multiple services, offices and commercial business which almost do not exist today. There are, as a huge need to increase the density of enterprises in the new neighborhoods of Lima. And neighborhood businesses generate employment in the neighborhoods (Infante, 2012). Productive cities are integrated mainly by micro, small and medium-sized enterprises. But, we sustain that Gamarra is a nascent cluster, such as product - inter alia - of the gap between small businesses big business and structural vulnerabilities that arise enterprises in our country, Gladys Triveño comment.

Presentamos, a continuación los aspectos preliminares, con el fin de enfatizar la importancia de las ciudades productivas integradas por micro, pequeñas y medianas empresas, señalando a Gamarra como un cluster incipiente.

\section{LAS CIUDADES PRODUCTIVAS EN EL PERÚ}

Desde la década de los 90 es indiscutible la evolución de Gamarra hacia un "clúster textil" relativamente más cohesionado; internamente la organización del complejo se presenta aparentemente fuerte, con una impresionante actividad comercial y productiva, pero que impide observar las carencias que obstaculizan su transformación. La problemática no culmina con la elección de Gamarra como ciudad productiva, en 2006. Sostenemos que no solo fue una decisión apresurada sino equivocada, porque a pesar del gran esfuerzo de sus asociados aún no cumple con los criterios básicos de una autentica ciudad productiva.

\section{CIUDADES PRODUCTIVAS}

Se trata de ciudades formadas por conglomerados de micro, pequeñas y medianas empresas, cuyo objetivo en las políticas públicas es generar empleo, en barrios y municipios, eliminando paulatinamente los cinturones de miseria urbanos y rurales.

Una primera impresión sobre la realidad de nuestro emporio textil nos presenta una amplia zona de edificios vetustos en su mayoría, y otros edificios relativamente modernos, con una actividad febril en todas sus calles repletas de personas, en medio de un ruido verdaderamente infernal. Esta apariencia de caos comercial y peatonal nos explica que Gamarra posee 14,000 establecimientos, 10,000 empresas de confecciones, comercio y servicios, 60,000 empleos entre empresarios y trabajadores, 150 manzanas de extensión, $800 \mathrm{mi}$ llones de dólares en ventas anuales y que paga 140 millones de dólares en impuestos. Y que posee un crecimiento de $17 \%$ anual, entre 1970 -1995, mientras que Perú solo creció $2.1 \%$.
Estas características realmente sorprendentes no son suficientes para su designación como ciudad productiva, en tanto sea un clúster incipiente, carente de reforzar la adquisición de insumos de calidad, resolver sus problemas de producción con servicios sistemáticos de asistencia técnica, desarrollar un plan de diseños y colecciones de temporada, servicios múltiples de "patronaje", tallas, productividad, que en su conjunto eleven la situación competitiva de Gamarra y la transformen en un auténtico conglomerado articulado.

La ausencia de una gestión empresarial moderna y la carencia de programas dinámicos de capacitación promovidos por sus directivos de vanguardia inciden en la naturaleza incipiente del clúster.

\section{Qué son los clústers}

La palabra refiere a un grupo de cosas similares posicionadas, o que ocurren en proximidad. En el ámbito productivo, se trata de una concentración geográfica de industrias por ventaja de desempeño. El clúster es denominado por Porter como el diamante de la competitividad.

A propósito de la trascendencia de las cadenas productivas y la insuficiencia de la explicación de la teoría de las ventajas competitivas de un país dado, León Martín Cabello sostiene que:

"De manera histórica, la competitividad de un país se había explicado a través de la teoría clásica de las ventajas competitivas, la cual pone el énfasis exclusivamente en la abundancia de recursos naturales y factores de producción. La competencia de los mercados es imperfecta; sus actores -empresas y Estados- actúan estratégicamente afectando los flujos comerciales y el desarrollo de los países. 
Las empresas deben tender a mejorar su cadena productiva, desarrollar tecnologías de proceso propias, diferenciación de productos, reputación de marca, relaciones y servicios a los clientes, entre otros".

Ahondando sobre la trascendencia, formación y evolución de las cadenas productivas, distintos enfoques teóricos intentan responder las causas de la formación de los clústeres. Stumpo, nos indica los más importantes:

- La teoría de localización y de geografía económica hace hincapié en el peso relativo del costo de transporte en el costo final, lo que explicaría por qué algunas actividades suelen ubicarse preferentemente cerca de los recursos naturales, otras se localizan cerca de los mercados que van a abastecer, en tanto que otras pueden establecerse en cualquier lugar.

- La teoría de los encadenamientos hacia atrás y hacia delante nos muestra cómo y cuándo la producción de un sector es suficiente para satisfacer el umbral mínimo o escala mínima necesaria para hacer atractiva la inversión en otro sector que este abastece (encadenamientos hacia atrás) o procesa (hacia adelante).

- La teoría de la interacción y los distritos industriales, establece la interacción que da lugar a juegos repetitivos que elevan la confianza y reducen los costos de transacción y de coordinación. Se acelera la difusión del conocimiento y la innovación, lo que es un bien social internalizado por el conjunto de empresas en el distrito. La interacción intensa en una localidad genera derrames tecnológicos y economías externas y de escala para el conjunto de empresas del distrito, que no podrían ser internalizados de estar cada empresa interactuando con las otras a grandes distancias.

- El modelo de Michael Porter sostiene que la diversidad e intensidad de las relaciones funcionales entre empresas explican la formación de un complejo productivo y su grado de madurez. Estas relaciones se refieren a la competencia entre empresas de la misma actividad, las relaciones con proveedores de insumos y factores especializados. Porter analiza el origen de la competitividad de las naciones con el objetivo final de desarrollar un marco conceptual para orientar a los empresarios en la toma de decisiones, como para la formulación de políticas industriales orientadas a promover el empleo y el desarrollo.

\section{Una ciudad andina}

Gamarra carece de un modelo de conglomerado o clúster articulado con patrones unificados e integrados de producción, marketing, administración y logística. Carece de unificar criterios corporativos que orienten a las mipymes debido a su fragmentación y el predominio de sus intereses particulares.

La insuficiencia de la legislación vigente, como la Ley 28015 y el D.L. 1086 no permitiría estimular y apoyar la asociatividad, las cadenas productivas y, por ende, el clúster en Gamarra. A propósito de la naturaleza monopólica de la producción textil basada en la explotación intensiva del algodón en los valles de la costa, identificamos grandes grupos económicos como el grupo Romero en las zonas de mayor explotación algodonera, en Piura, Chincha y Lima. Este es un punto importante de la investigación por su vinculación a los canales de comercialización nacional e internacionales relativas a los costos. Nos referimos al acopio de materia prima y cómo llegan estos volúmenes a Gamarra. Unida a esta variable, es preciso anotar la seria incidencia de dumping en el ingreso de materia prima y productos terminados por debajo de los costos nacionales desde Asia, la India particularmente.

Otro ángulo de la investigación es la clara insuficiencia de marcas o un mayor desarrollo del branding en Gamarra, lo cual es un inocultable factor de mengua de competitividad para nuestra producción textil, concomitantemente con la conquista de mejores oportunidades. Excepto Topitop, Pieers, Caman, Kansas, Tayssir, -una golondrina no hace el verano- no existe una marca posicionada "Gamarra Perú" que podría eventualmente reforzar la posición competitiva del clús-

1 VILLARÁN, Fernando (1998). Capitalismo popular, pasión y gloria de la pequeña empresa en el Perú. En: Edición del Congreso de la República del Perú, 1998.

2 CABELLO, León Martín (2003). Apertura comercial y productiva de México, 2003.En: Publicación del Premio Alejandrina, julio del 2003, México.

3 STUMPO, G (1996).Encadenamientos, articulación y procesos del desarrollo industrial. En: Revista de la CEPAL, setiembre de 1996, Santiago de Chile, CEPAL. 
ter en EE.UU., primer destino de exportación, o Venezuela, segundo destino de exportación textil peruano, y otros destinos de exportación tan o más competitivos que el aludido. (Klaua, Martín y Daneliuc, Estevan. Entrevista Día_1 del 8-3-2010).

\section{EXPERIENCIAS EXITOSAS DE CIUDADES PRODUCTIVAS}

Existen múltiples indicios de la existencia de ciudades productivas a nivel global aunque con escasa y dispersa bibliografía de análisis e investigación sobre ellas. Consignamos a continuación las experiencias relevantes de clústeres y la formación de consorcios exportadores en Italia y España, que constituyen elementos básicos para la organización de estas ciudades.

El crecimiento de un clúster regional suele superar al de un país, pues se trata de alineamientos empresariales hacia objetivos comunes, centrados en la competitividad.

\section{ITALIA}

Los consorcios exportadores nacieron en Italia en 1965, por la necesidad de las pymes de unirse y llegar a los mercados externos. Operan en forma autónoma o sirviéndose de la colaboración del Instituto de Comercio Exterior (ICE) y de las Cámaras de Comercio Italianas en el extranjero.

\section{Niveles de crecimiento}

El ritmo de crecimiento de un clúster regional suele superar el del país que lo acuna en su conjunto. Ya que se trata de alineamientos empresariales hacia objetivos comunes, centrados en la competitividad, y constituidos a través de redes de confianza que consolidan regiones económicas prósperas.

Italia tiene la particularidad de tener las pequeñas industrias concentradas en pequeñas ciudades, dando lugar a que la tradición y proximidad geográfica acaben creando una red de confianza que facilite la asociación regional de las empresas (aglomeraciones o clústeres) y la formación de consorcios exportadores.

De acuerdo a la legislación italiana, el consorcio debe estar formado al menos por ocho pymes, (cinco si la empresa tiene sede al sur de Italia) las cuales según el sector, la ubicación y las finalidades previstas determinarán el tipo de consorcio.
El Estado, a través de distintas leyes, financia y contribuye al crecimiento de los consorcios de exportación. Una de las principales leyes, la 83/89, otorga desde hace 30 años un aporte financiero a los consorcios, que varía de acuerdo al balance que presente cada grupo.

\section{La gran experiencia italiana}

- Sexto exportador mundial.

- Primer exportador per cápita.

- Décimo primer exportador en inversiones.

- Un tercio de las exportaciones son realizadas por distritos industriales.

Podríamos resumir que los factores de competitividad son la creatividad, la innovación, la flexibilidad, la confiabilidad, los recursos humanos, un insólito culto al producto y a la marca.

\section{España}

La figura del "consorcio exportador" desempeña un papel destacado en la economía española, donde un gran número de empresas son de reducida dimensión y escasa experiencia internacional. En los últimos años, el Gobierno español como las comunidades regionales y entidades privadas han puesto en marcha exitosos programas de apoyo en temas vinculados a la exportación. Así nacieron los "grupos exportadores", al amparo de los programas del Instituto de Comercio Exterior de España (ICEX), dependiente del Ministerio de Economía.

Los requisitos para ser parte de un "consorcio exportador" exigen personalidad jurídica propia e independiente de las empresas que lo componen, estar al día en el cumplimiento de sus obligaciones fiscales, de seguridad social y en materia laboral. Deben estar formadas por un mínimo de cuatro empresas fabricantes españolas, que por razones justificadas pueden reducir sus miembros a solo tres. Incluso si el consorcio se ha establecido en el exterior, el número mínimo también es de tres.

Además de contar con un gerente imparcial y con dedicación exclusiva, presentar un plan de actuación en el exterior y de viabilidad económicofinanciera, ambos a cuatro años desde el inicio. Dada la importancia del papel del coordinador en la formación y dinámica del grupo, las Cámaras de Comercio en cada área autónoma participan en la preselección del candidato- aunque sin las empresas-y son las que toman la decisión final, 
y es ICEX quien paga el 50\% de su sueldo en la etapa inicial del proyecto ( Gálvez, César; Vicuña, Carla. 2004)

\section{CIUDADES PRODUCTIVAS COMO MODELO DE DESARROLLO}

La investigación nos parece relevante porque proponemos un modelo de desarrollo económico basado en ciudades productivas y porque las decisiones de asociatividad no se contradicen con la búsqueda de un verdadero conglomerado consorcio-productivo, que también posee las auténticas características superiores a un clúster. La evidencia empírica para el caso peruano señalaba que la gran mayoría de ellas escogía competir individualmente en razón de la carencia de esta cultura de asociatividad, particularmente.

A contrapelo de su elección individual, sostenemos que en la gran mayoría de los casos no cuentan con las capacidades necesarias para encarar el reto de crecer individualmente en el mercado interno. Mucho menos se encuentran en condiciones de exportar competitivamente. En este constructo de finalidad, reiteramos la justificación práctica de nuestra investigación que busca:

- Promover una política de agresiva competitividad, para lograr un mejor posicionamiento de los productos textiles en el ámbito mundial a través de los clústeres.

- Aprovechar las ventajas competitivas del algodón peruano y la mano de obra especializada que genera gran demanda a nivel internacional constituyéndose en un potencial exportador por excelencia.

- Prelanzamiento de un modelo nacional con potencial exportador a nivel nacional e internacional mediante marcas de bandera.

- Lograr el éxito de una cruzada cultural, que genere cambios proactivos en el comportamiento organizacional de los agentes que forman parte del sistema productivo y comercial, porque la unión hace la fuerza.

A propósito del concepto de ciudades productivas carecemos, para la investigación, de mayores referencias bibliográficas que puedan relevarse en términos de replicabilidad práctica, así como en términos de la epistemología de la investigación. La escasez y dispersión de teoría sobre modelos de ciudades productivas es la principal limitación de la investigación en países como el nuestro, con economías emergentes y en vías de desarrollo.

El programa denominado "Ciudades Productivas", se inició en el gobierno del presidente Alejandro Toledo, en el año 2005 y su objetivo primigenio era reemplazar los cinturones de marginación y miseria existentes en zonas periféricas de las urbes - donde por lo general inician sus actividades las mipymes- por cinturones de vivienda productivas organizados en complejos y sistemas integrados,.

"Las ciudades productivas son modelos de desarrollo socioeconómico que involucran un plan integral que establece la asociatividad empresarial y la generación de redes comerciales". (Lemor, David 2005).

La ciudad productiva de este distrito fue el inicio de este proyecto para las microempresas. Hasta la fecha, refirió en el año 2005, son más de 20 mil pymes generadas en estas ciudades productivas.

"Este es el semillero de un sistema autogestionario que no solamente involucra el ordenamiento de las empresas y su producción, sino que también se preocupa por la seguridad ciudadana en las zonas productivas. Importante proyecto al que se sumaron otras seis ciudades: Gamarra (Lima), Los Olivos (Lima), El Porvenir (Trujillo), San Jerónimo de Tunán (Junín), Concepción (Junín) y Ate" (Lemor, David 2005).

Esta iniciativa gubernamental se reiteró el 16 de agosto del 2006, con la intervención del ingeniero Rafael Rey en la presentación del Programa de Articulación Productiva-Comercial en Villa el Salvador (Rey, Rafael 2007).

Los peruanos hemos desarrollado la iniciativa, la originalidad como consecuencia de no tener las ventajas que otras personas en otros países sí tienen. Y ese es un capital para los peruanos y el Parque Industrial de Villa El Salvador, y en general el empuje de nuestros pequeños, micro y medianos empresarios demuestra esto: que los peruanos no somos menos que nadie; al contrario, hemos desarrollado una habilidad innata como consecuencia de que no nos ha sido fácil hacer estas cosas, y eso es algo que debe ser el orgullo para todos los peruanos, y eso es lo que muestra en concreto el Parque Industrial de Villa El Salvador. 


\section{Importaciones subvaluadas}

Uno de los principales problemas que enfrentan las industrias locales son las importaciones de materias primas subvaluadas. Esta mercancía puede alcanzar un rango de subvaluación de hasta $70 \%$. Y para muestra basta un botón, que importado reduce su costo a una ínfima parte relacionada con el precio ofrecido por los proveedores locales.

Asimismo la reiteración del alcalde del Distrito de Villa el Salvador (Zea, Jaime. 2005):

El parque industrial no solo ha demostrado ser un núcleo de desarrollo empresarial, sino que muestra, también, los resultados que se pueden obtener con trabajo concertado. Además de impulsar el crecimiento de nuestro distrito como ciudad productiva, contamos con proyectos destinados a consolidarlo como tal. Mejoraremos los dos pasos a desnivel de las avenidas El Sol y Juan Velasco, lo que hará más fácil el acceso al parque industrial. Asimismo, se ha previsto reordenar a los comerciantes y mejorar el entorno ambiental".

Un avance innegable es que Gamarra posee varias organizaciones empresariales, pero unitariamente reunidas en la Coordinadora de Empresarios de Gamarra (Villarán, Fernando 1998).

Otro avance relevante es que nuestro emporio textil es agenda multisectorial por institucionalizar su reciente nombramiento como la segunda ciudad productiva en Lima, después de Villa El Salvador designada como la primera ciudad productiva en octubre pasado.

\section{LA PRODUCCIÓN TEXTIL PERUANA 1990-2007}

La industria textil peruana ha sido muy exitosa en el mercado de las confecciones. Pocas industrias textiles de otros países han logrado mantener un ritmo de exportaciones crecientes en este segmento, como lo ha hecho la del Perú. El sector se caracteriza por lo siguiente:

- Precios internos afectados por subsidios.

- Producción nacional con tendencia decreciente.

- Importaciones en aumento.

- Excelente calidad de la fibra empleada.

- Baja tecnología.

- Precios del mercado reconocen calidad.

- Demanda local en crecimiento.
Según el mitinci, 34 empresas generan más del $70 \%$ del valor agregado de la producción de hilados y tejidos. En las industrias de hilados y textiles, según el Ministerio de Industrias y Aduanas, las empresas grandes -con 201 trabajadores o más- son el $1 \%$ de todas las empresas registradas formalmente en ambas industrias y concentran el $26 \%$ de la mano de obra empleada y el $94 \%$ del valor exportado por ambas industrias. En la industria de confecciones, las empresas grandes son también el $1 \%$ de todas las empresas registradas formalmente en la industria y emplean el $8 \%$ de la mano de obra de esta industria y el $82 \%$ del valor exportado por ella.

Aunque prácticamente solo las empresas de mayor tamaño son las que exportan directamente, es muy frecuente que mediante la modalidad de subcontratación las grandes empresas compren productos a las medianas (21 a 200 trabajadores) y pequeñas empresas (11 a 20 trabajadores). Estas, a su vez, contratan con frecuencia a las microempresas (1 a 10 trabajadores), tanto para la producción requerida para el mercado local como para la exportación.

El 70.5\% de las empresas de las industrias de hilados y tejidos y el 78\% de las empresas de la industria de confecciones se localizan en Lima y Callao. En el 2010 se han registrado 24,000 empresas en el rubro textil, y que emplean a 400,000 personas, directamente.

\section{REDES EMPRESARIALES EN EL CONGLOMERADO DE CONFECCIONES DE GAMARRA}

Comenzaremos dando una breve reseña de la importancia que tiene este aglomerado textilcomercial: Gamarra continúa siendo el principal centro de abastecimiento y comercialización del rubro textil, preferido por los comerciantes minoristas y mayoristas asiduos básicamente por tres aspectos fundamentales:

1. Ofrece precios realmente competitivos y económicos que les permiten sostener un margen de ganancia "respetable", acorde al nivel de expectativas en un mercado golpeado por la crisis económica como el nuestro.

2. Permite acceder a variedad de mercadería principalmente en el rubro textil, lo que implica diversidad de diseños, prendas, calidades, colores y tallas. 
3. Las confecciones están a la par de los nuevos estilos de moda.

Gamarra tiene 17,000 establecimientos distribuidos en 125 galerías comerciales (más de dos pisos) y en 800 casas comerciales (casas acondicionadas como comercio). Trabajan aproximadamente 60,000 personas. Se dice que en Gamarra se mueven 800 millones de dólares al año, más o menos un promedio de 4,000 dólares de venta mensual por tienda. El crecimiento anual de Gamarra aproximadamente ha sido de 500 establecimientos.

El proyecto Redes Empresariales en el Conglomerado de Confecciones de Gamarra está siendo mayormente difundido y ejecutado en la actualidad por la Comisión de Promoción de la Pequeña y Micro Empresa (PROMPYME), definiendo una red empresarial como una alianza estratégica entre un grupo determinado de empresas que comparten objetivos comunes de negocio y trabajan juntas para alcanzarlos.

\section{Redes empresariales}

El ambiente de negocio virtual ha generado nuevas maneras de los empresarios para relacionarse, como redes empresariales por Internet, y estrategias para conformar procesos más dinámicos en toda la cadena

Una ciudad productiva es un modelo de desarrollo socioeconómico de comercios que representan la cooperación de las mipymes para tener mayor competitividad.

\section{INDICADORES DE LA INVESTIGACION}

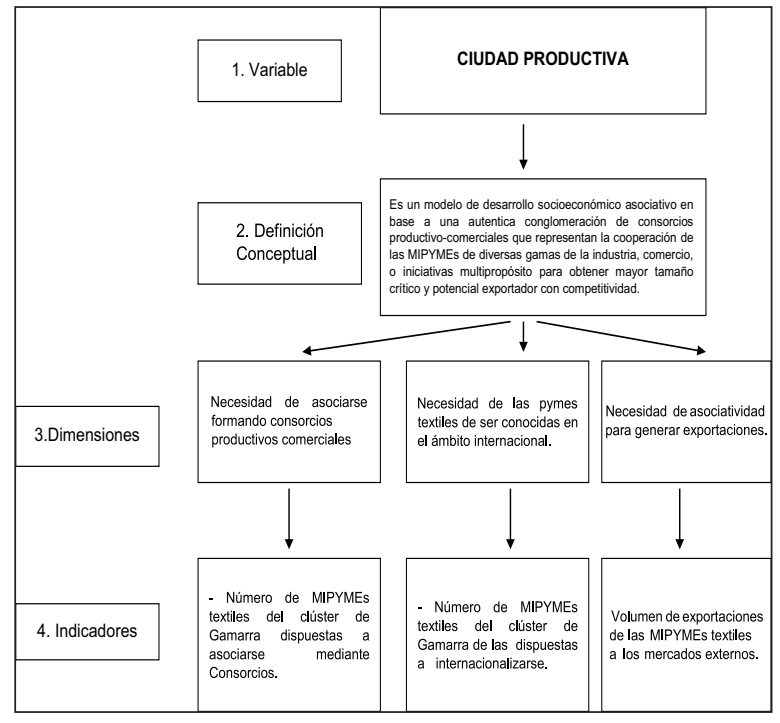

Fuente.- Elaboración propia

\section{Perú superará a Chile}

Sin duda, la economía de Perú superará a la de Chile dentro de los próximos 38 años y se situará en el privilegiado lugar 26 de un conjunto de 100 economías más grandes del mundo, reveló el estudio "Mundo en 2050", elaborado por el banco HSBC.

Actualmente, de acuerdo a sus propios datos, que corresponden hasta 2010, Perú ocupa el puesto 46 con un PIB de 85 mil millones de dólares (del año 2000), mientras que su vecino del sur, Chile, se sitúa en el lugar 44 .

\section{ANÁLISIS FODA DEL SECTOR TEXTIL}

Ensayamos a continuación el análisis estratégico del sector:

\section{FORTALEZAS}

Mejoramiento de la imagen del Perú a nivel internacional, basado en la estabilidad y crecimiento económico con respecto a sus países vecinos.

Cercanía al mercado americano, a los países de la comunidad andina y Chile. La rapidez de aprovisionamiento del producto es un factor apreciado por los importadores. Para vestir nacionales, pueden competir mejor con los países asiáticos que se encuentran más alejados.

El Perú tiene prestigio internacional como productor de algodón de fibras de buena calidad; este es un factor básico que coloca al Perú en una posición ventajosa respecto otros países productores de textiles de algodón.

En la costa existen regiones apropiadas para el cultivo de algodón por sus condiciones de calor, temperatura y luminosidad; pudiendo aprovechar las zonas áridas porque el cultivo requiere poca agua y no es muy exigente en calidad de suelos. Además el agricultor nacional ha cultivado algodón desde tiempos ancestrales, por lo que se encuentra debidamente capacitado en el manejo agronómico de este cultivo.

En el mercado internacional, los precios de algodones de fibra larga y extralarga están mucho mejor cotizados que los algodones de fibra corta. El 90\% del comercio del algodón mundial corresponde a algodones de fibra corta y media; sin embargo, el Perú produce dos variedades que son de la mejor calidad, de fibra larga y extralarga: el tanguis y el pima respectivamente. 
Los empresarios de textil y prendas de vestir cuentan con 15 años de experiencia exportadora, por lo que han adquirido un nivel de "knowhow" en comercialización y producción, que les permite competir apropiadamente en el mercado internacional. Además existe toda una red de subcontratistas y proveedores de accesorios capaces de cubrir los aumentos de demanda.

Se ha conformado el Instituto Peruano del Algodón (IPA), donde participan productores de algodón, desmotadores, hilanderos, textiles y confeccionistas; es decir, toda la cadena productiva, con el propósito de desarrollar activamente variedades de semilla de algodón competitivas internacionalmente en productividad y calidad de fibra.

\section{DEBILIDADES}

- Falta de una política adecuada de apoyo para las pyme exportadoras.

- Falta de información y/o capacitación.

- Dificultades de financiamiento.

- No existe innovación tecnológica.

- Concentración de exportadores en un número reducido de empresas.

- Altos costos de transporte, servicios, y sobrecostos laborales.

- La importación de ropa usada y las prácticas de contrabando, el subconteo, la subvaluación.

- Es imperativo exportar para poder competir. Para ello es primordial una alianza entre empresarios para alcanzar esta meta, así lo deben entender los microempresarios.

\section{OPORTUNIDADES}

- Apertura comercial.

- Liberación arancelaria.

- Acuerdos y convenios internacionales.

- Variedades de tejidos.

- No se explota trabajar con los empresarios norteamericanos a través de contratos de maquila.

- Desarrollo del clúster textil a nivel mundial.

- Generar empleo.

\section{AMENAZAS}

- Políticas que permitan importar masivamente productos textiles a precios por debajo de los costos nacionales o dumping (Asia).
- Competidores textiles (China, México).

- Factores que hacen peligrar la producción de algodón: productividad mundial, subsidios, Fenómeno de El niño, calentamiento global y cambio al cultivo del maíz amarillo.

\section{CONCLUSIONES}

Con la primera prueba de hipótesis llegamos a la conclusión de que los microempresarios de Gamarra no se muestran indiferentes ante una posible alianza estratégica llamada Red Empresarial. Esto lo corroboran los datos estadísticos, un $80 \%$ de la muestra estuvo de acuerdo en aceptar dicha opción (red empresarial) como una alternativa para mejorar su negocio. Y así también lo constata la prueba de hipótesis realizada.

Este resultado tiene mucho que ver con el reto que tiene la industria de confecciones: exportar. La pequeña empresa en el país tiene que desarrollarse; ninguna ventaja es permanente. Son tantas las empresas que existen que, a pesar de que aumentan las ventas, disminuyen los ingresos: si antes vendías 100 , lo repartías entre 20 ; ahora vendes 120 y lo repartes entre 60 . El imperativo es exportar. Y para esto resulta primordial una alianza entre empresarios para alcanzar esta meta; y los microempresarios así lo comprenden. Al año 2010 se han registrado 23,400 empresas textiles que emplean directamente a 400,000 personas. El impacto de la crisis externa no ha mellado considerablemente en el sector y las previsiones son relativamente halaguieñas. Las exportaciones se redujeron en $29 \%$ con relación al 2008 (El informe, pág. 12 ¿Quo Vadis? Día_1 El Comercio. 8-3-10).

Con un diseño del modelo de desarrollo socioeconómico asociativo de conglomerado industrial comercial, se podría lograr que las Mipymes textiles de Gamarra se transformen en una auténtica ciudad productiva de naturaleza inclusiva y desarrollen su potencial exportador con competitividad con base en la asociatividad empresarial y la generación de redes comerciales.

\section{REFERENCIAS BIBLIOGRÁFICAS}

BECATTINI, Giacomo (2005). "Qué son los Distritos Industriales". <www.eumed.net. www.dialnet.unirioja.es/servlet/fichero. articulo?codigo:59802...0.>

BERNAL, César Augusto (2006). "Metodología de la investigación”. Ed. Pearson, 2006. 
Cavani Grau, Carlos (2007). “Consorcios, estrategias integrativas de marketing para el desarrollo autosostenido de las MIPYMEs. Caso: MIPYMEs metalmecánicas del Parque Industrial de Villa el Salvador". Tesis Universidad Nacional Mayor de San Marcos. Presentado en el libro "El Consorcio, Experiencias Asociativas de Marketing en Villa El Salvador" (2013). En: Fondo Editorial de la UNMSM, 2013.

Chevalier y Toledano (1978). "Las Cadenas Productivas” < http://agrobanco.com.pe/cad-prod. htm. $>$

Durufle; Fabre, R; Young, J; (1988). "Las Cadenas Productivas” < http// agrobanco.com.pe/ cad_prod.htm.1988.>

Gálvez, César; Vicuña, Carla (2004). Condiciones para la formación de Consorcios de exportación en las Pymes confeccionistas de Gamarra en el marco del ATPDEA. Tesis, URP-Perú. En: <www.cybertesis.com>

Gonzales, Julia (2006). "Redes de la informalidad en Gamarra”. En: Editorial Universitaria de la Universidad Ricardo Palma, 2006.

Hernández Iglesias, José (2005). “Hacia una epistemología de la administración para pymes en un mundo globalizado". <www.google. com>

Lemor, David (2005). Lanzamiento del proyecto de Ciudades Productivas en Villa El Salvador. En: "Propuesta de un Modelo de Ciudad Productiva enlazada al mercado global para el Desarrollo del Conglomerado Textil de Gamarra” En: Tesis Doctoral de Cavani Grau, Carlos, 2012, pág. 35. Universidad Ricardo Palma, 2012.

Marshall, Alfred (1890). "Principles of Economics". $<$ www.wikipedia.com>

Matos Mar, José. (2004). "Desborde popular y crisis del Estado”. Ediciones del Congreso de la República, 2004.

Montigaud, Jean (1995). "Las Cadenas Productivas" < www.mapa.es/ministerio/pagsbiblioteca/.../agri 1995.756.595.597.pdf

Regalado, Rafael (2007). Las Mipymes en Latinoamérica, Edit. EUMED, España, 2007. <www. eumed.net>
Rey, Rafael (2007). Presentación del Programa de Articulación productiva-comercial en Villa El Salvador. En: "Propuesta de un Modelo de Ciudad Productiva enlazada al mercado global para el Desarrollo del Conglomerado Textil de Gamarra” En: Tesis Doctoral de Cavani Grau, Carlos, 2012, pág. 35. Universidad Ricardo Palma, 2012.

Sánchez Palacios, Humberto (2004). "Gamarra, hacia la conquista del mercado exterior". En: Boletín publicado por la Cámara de las Pymes Exportadoras del Perú - CAPYMEX, 2004.

Rosales, Ramón (1997). "La Asociatividad como estrategia de fortalecimiento de las PyMES”. En: Publicación de la Universidad de Texas, setiembre de 1997.

Sociedad Alemana de Cooperación Tecnica (1997). $<$ www.google.com>

Zea, Jaime. (2005).Ceremonia del Consejo Andino de Autoridades Municipales. En: "Propuesta de un Modelo de Ciudad Productiva enlazada al mercado global para el Desarrollo del Conglomerado Textil de Gamarra" En: Tesis Doctoral de Cavani Grau, Carlos, 2012, pág. 36. Universidad Ricardo Palma, 2012.

\section{REFERENCIAS DE LA LECTURA}

Villarán, Fernando (1998). “Capitalismo popular, pasión y gloria de la pequeña empresa en el Perú”. Ediciones del Congreso de la República, 1998.

Cabello, León Martín. (2003) "Apertura productiva y comercial de México”. Publicación del Premio Alejandrina, julio del 2003, México.

Stumpo, G. (1996). Encadenamientos, articulación y procesos del desarrollo industrial. Santiago de Chile, CEPAL. En: Revista de la CEPAL, setiembre de 1996, Santiago de Chile, CEPAL.

Klaua, Martin y Daneliuc, Estevan (2010). En: Entrevista "Día 1" del 8-3-2010, suplemento del diario El Comercio, Lima- Perú. 
